Archives

2| 1988

Varia

\title{
L'essor de Budapest à l'époque dualiste
}

\section{Karoly Voros}

\section{OpenEdition}

Journals

\section{Édition électronique}

URL : http://journals.openedition.org/ccrh/2963

DOI : $10.4000 /$ ccrh.2963

ISSN : $1760-7906$

\section{Éditeur}

Centre de recherches historiques - EHESS

\section{Édition imprimée}

Date de publication : 15 avril 1988

ISSN : 0990-9141

\section{Référence électronique}

Karoly Voros, "L'essor de Budapest à l'époque dualiste ", Les Cahiers du Centre de Recherches Historiques [En ligne], 2 | 1988, mis en ligne le 13 avril 2009, consulté le 21 avril 2019. URL : http:// journals.openedition.org/ccrh/2963 ; DOI : 10.4000/ccrh.2963

Ce document a été généré automatiquement le 21 avril 2019

Article L.111-1 du Code de la propriété intellectuelle. 


\title{
L'essor de Budapest à l'époque dualiste
}

\author{
Karoly Voros
}

1 En 1849, les forces militaires conjointes des Habsbourg et des Romanov écrasèrent l'insurrection hongroise. Pourtant elles ne purent éliminer les acquis de la révolution bourgeoise qui défendait l'insurrection : la Hongrie s'était engagée irrévocablement dans la voie conduisant au développement capitaliste.

2 Après 1849, le rôle joué par Pest-Buda dans ce développement apparait de plus en plus important. Dès le début des années 1860, le commerce des produits agricoles, bénéficiant de la forte concentration du réseau ferroviaire, tenait une place prépondérante dans l'économie hongroise. Le capital accumulé était investi dans des secteurs et des entreprises industrielles qui échappaient à la concurrence de l'industrie autrichienne plus développée. Ainsi, à partir des matières premières du commerce de Pest, les commerçants bâtirent, du milieu des années 1850 jusque vers 1873, la plus grande industrie de la minoterie d'Europe. Vers la fin des années 1860, elle exportait jusqu'en Amérique du Sud. La distillerie connut aussi un développement considérable. Pour satisfaire aux besoins du pays en équipements destinés à l'agriculture et aux transports, un certain nombre d'entreprises métallurgiques, de fonderies et d'ateliers de construction mécanique furent créés : ainsi l'usine de Ganz, issue de la petite fonderie de fer fondée en 1845 et dont les roues de wagon, produites selon une technique brevetée de fonderie, étaient vendues jusqu'en Europe occidentale vers la fin des années 1860.

3 La population continuait à affluer de tout le pays vers ces villes (178 000 habitants en 1851, 187000 en 1857, 270000 en 1869). À Pest, 45000 habitants sur 200000 n'étaient pas originaires de la ville. L'aspect de la ville se transforma également: à la périphérie commencèrent à se former des zones industrielles; la régularisation du cours du Danube entre les deux villes fut engagée, ainsi que la construction des quais; les gares furent construites au terminus des lignes ferroviaires conduisant à Pest-Buda. De nouvelles rues furent tracées et les anciennes furent rénovées; le nombre des maisons de trois ou plusieurs étages devint plus important. Dans le style architectural, on vit apparaître, à côté du classicisme tardif, le romantisme et même l'éclectisme précoce. En 1856, une 
usine à gaz fut créée à Pest et on installa le réseau d'éclairage au gaz; en 1862, il en fut de même à Buda. En 1867, le premier grand service d'eau fut mis en place à Pest. En 1866, les premiers tramways furent mis en circulation; en 1878, on construisit dans le quartier du château un funiculaire à vapeur, et, en 1873 , le chemin de fer à crémaillère fut installé dans les collines de Buda afin de mieux les insérer dans le réseau de la circulation urbaine. La ville en croissance était plus que jamais le centre de la culture hongroise et du mouvement national hongrois, qui s'affirma de plus en plus ouvertement à partir de la fin des années 1850. Dans cette lutte ouverte, les Hongrois, devenus majoritaires à cette époque, étaient même soutenus par une partie de l'ancienne bourgeoisie allemande dont les intérêts n'allaient pas dans le sens d'une dépendance politique du pays.

4 Après le compromis austro-hongrois de 1867, la classe dominante hongroise, poussée par des raisons aussi bien politiques qu'économiques, s'efforça d'accélérer le développement de la capitale : une loi sur le développement urbain fut adoptée et pour diriger les travaux on créa le Conseil des Travaux Publics, indépendant du Conseil Municipal, sous la direction du Premier Ministre. En 1872, à l'issue d'un concours international, un plan d'aménagement de la capitale fut élaboré. En 1872, Pest, Buda et Obuda furent réunies en une seule ville, légalement et juridiquement; en 1873, ces trois villes ne formaient plus effectivement qu'une seule ville.

5 La bourgeoisie de la capitale, qui se réjouissait du compromis austro-hongrois et de l'unification des villes, dut pourtant faire face aux premières tentatives d'organisation de la classe ouvrière de Budapest. En 1868, fut créée l'Association générale des Ouvriers sous l'influence des idées de Lassalle; mais les internationalistes marxistes y occupèrent rapidement une place importante, essentiellement sous l'influence de la Commune de Paris. Dès 1870 et 1871, on assista à des grèves imposantes et à des manifestations de rues; les ouvriers fêtèrent les héros de la Commune de Paris. Le procès intenté par le gouvernement contre les dirigeants de l'Association des Ouvriers fut un échec mais il eut pour résultat d'empêcher la transformation de l'Association en un vrai parti politique; le parti ouvrier en formation fut immédiatement dissous par le gouvernement; ainsi, pendant des années encore la classe ouvrière devait rester dépourvue d'organisation et de représentation politique.

6 Au moment de l'unification, la capitale était déjà, sans aucun doute, le centre le plus important de la vie économique du pays. De 1873 à la fin de la première guerre mondiale, l'extension du réseau ferroviaire et sa centralisation à Budapest, qui traduisait une tendance délibérée de la politique gouvernementale, contribuèrent à accroître l'importance de la ville tant sur le marché intérieur en formation que dans son rôle d'intermédiaire pour le commerce d'Europe centrale et des Balkans. Pour accueillir les marchandises qui transitaient par la ville ou y étaient stockées temporairement, d'immenses silos à blé équipés d'élévateurs modernes furent construits. Au début du $\mathrm{xx}^{\mathrm{e}}$ siècle, les voies ferrées arrivaient à Budapest par deux ponts de chemin de fer, en provenance de onze directions et le système de tarifs des voyageurs instauré à cette époque rapprochait les régions les plus éloignées de la sphère d'attraction de Budapest. La construction du canal de la Porte de Fer augmenta de façon notable les capacités de la navigation danubienne. Le système des gares de marchandises et de voyageurs fut mis en place, inséré dans la ligne de ceinture qui entourait la ville, et on envisagea la construction d'un grand port danubien pour la navigation. La Bourse de Budapest devint le centre du commerce du blé pour tout le pays. A Köbanya fut créé le centre de commerce des porcs le plus important d'Europe centrale et orientale. 
7 Cette centralisation du commerce et des transports contribua encore à l'accumulation capitaliste par la bourgeoisie. Les banques et caisses d'épargne de la capitale, remarquablement consolidées, devinrent bien vite une base et un intermédiaire pour l'entrée en Hongrie des capitaux allemands, autrichiens et français. Au début du xx siècle, les cinq institutions de crédit les plus importantes de Budapest $\mathrm{y}$ concentraient près de $60 \%$ des capitaux qu'elles possédaient dans l'ensemble du pays; grâce à l'extension de leurs filiales, elles jouèrent un rôle de plus en plus important dans le crédit des provinces. Grâce à leur initiative et à leur rôle d'intermédiaire à l'égard des capitaux occidentaux, les banques les plus importantes de Budapest commencèrent à s'introduire également dans les Balkans.

8 A partir des années 1880 , le développement industriel, provisoirement suspendu par l'échec des tentatives européennes de création d'entreprises, s'intensifia de nouveau, parfois interrompu lors de crises temporaires. En raison du recul du commerce dans certains secteurs, imputable à l'indépendance économique croissante des pays balkaniques, le rôle de l'industrie de fabrication dans la vie économique et sociale de la capitale devint de plus en plus important. Dans les dix dernières années du XIX ${ }^{\mathrm{e}}$ siècle, l'influence des banques dans le secteur industriel contribua tout particulièrement à son développement ; ainsi, les cinq grandes banques de Budapest contrôlaient $47 \%$ du capital des sociétés anonymes industrielles du pays.

Budapest, par tradition sa plus grande place marchande fut, dès l'origine, le plus grand marché financier du pays, tout en conservant le premier rôle dans le développement industriel de la fin du siècle. Le développement de l'industrie de fabrication fut largement favorisé par la centralisation des transports; le Danube satisfaisait complètement les besoins en eau de la grande industrie. A partir des années 1880, le développement de l'industrie minotière se ralentit et elle perdit le premier rang qu'elle occupait dans l'industrie de la ville au profit des industries mécanique, chimique et électrique.

Dès le début $\mathrm{du} x \mathrm{x}^{\mathrm{e}}$ siècle, la mise en place du réseau des trains de banlieue entraîna la formation des banlieues. De grandes entreprises y furent rapidement créées; leur installation à distance de la métropole fut favorisée par l'absence des lourdes charges fiscales qui pesaient sur la capitale. Signalons, parmi les zones industrielles situées au Nord et au Sud de Pest, la grande usine métallurgique implantée dans la partie Nord de l'île Csepel et destinée surtout à produire du matériel de guerre. À la veille de la première guerre mondiale, le plus grand complexe industriel de Hongrie se situait à Budapest et dans sa périphérie. En 1910, 289 entreprises employant plus de cent ouvriers fonctionnaient dans la ville actuelle ; 17 d'entre elles comptaient plus de mille ouvriers.

11 Le fait qu'à cette époque Budapest soit resté le plus grand marché de main d'oeuvre du pays est l'une des causes et des conséquences de cette situation. Ce marché était alimenté en premier lieu par la population des grandes propriétés foncières, dont les chances d'ascension sociale étaient faibles, et par les Juifs venant des provinces tchèques, polonaises et autrichiennes: ils constituent pendant toute cette période, et de façon constante, un quart de la population de Budapest. Celle-ci qui comptait 270000 habitants en 1869, atteignit 930000 habitants en 1913; Budapest devint ainsi la huitième ville européenne. On dépassait déjà le million, si l'on inclut les immigrés habitant les banlieues. En deux générations, environ un million et demi de personnes étaient devenues des citadins de Budapest ; cette situation influença de façon décisive leur mode de vie, leur conscience sociale et leur comportement politique. Cette population, venue presque toute entière d'un milieu rural, se transforma en un salariat industriel; le 
producteur de vivres indépendant devint acheteur et consommateur ainsi que locataire de son logement. Dans le même temps, et pendant une ou deux générations, s'engagea la magyarisation des immigrés dont un pourcentage élevé ne parlait pas le hongrois. Tout cela comportait des risques constants de conflits sociaux, et le danger d'aliénation qui menace l'homme détaché de son milieu traditionnel.

L'urbanisation coordonnée et partiellement dirigée par le Conseil des Travaux Publics accompagna de façon plus ou moins heureuse cette évolution économique et cet accroissement de la population. Entre 1870 et 1910, le nombre des maisons doubla, passant de 9351 à 18035 , et le nombre des étages quadrupla atteignant le nombre de 35876 ; la proportion des maisons de trois étages et plus passa de $2,1 \%$ à $18,7 \%$; les rues et places principales furent construites conformément aux plans établis au début des années 1870 , dans le style éclectique et historisant, puis, à partir du début du $\mathrm{xx}^{\mathrm{e}}$ siècle, de plus en plus dans le style "moderne" dit "sécession". A l'occasion de l'achèvement du Grand Boulevard ou de l'avenue du Nouvel Apparat, des quartiers entiers disparurent. L'administration municipale elle-même dut quitter l'ancien hôtel de ville dans la Cité, démoli avec tout son quartier pendant la construction du pont Elisabeth, pour aller s'installer dans le Palais des Invalides acheté à cette intention. Des immeubles luxueux et des bâtiments publics représentatifs de l'État furent construits; dans les quartiers extérieurs en cours d'urbanisation, le long de petites rues tracées de façon à permettre la meilleure utilisation des services publics, s'élevaient les maisons des pauvres : des citéscasernes. Même dans le quartier historique du château, à Buda, qui fut laissé en grande partie intact, on édifia de nouveaux bâtiments : ainsi l'aile nouvelle du Palais royal fut construite en style néobaroque, l'église Mathias fut restaurée en style néo-gothique suivant les principes de Viollet-le-Duc, le Bastion des Pêcheurs construit autour de l'église fut édifié en style néo-roman; quelques bâtiments furent également construits pour le gouvernement. L'exposition organisée pour le millénaire de l'occupation du pays par les Hongrois (896) puis l'agrandissement du Jardin zoologique et la construction du quartier des musées embellirent le Bois de Ville.

Pour améliorer la circulation dans la ville, on construisit trois ponts sur le Danube entre 1876 et 1912. Parmi eux, le pont Elisabeth est resté pendant longtemps le plus grand pont suspendu d'une seule portée existant en Europe. En 1912, le réseau des lignes de tramways, peu à peu électrifié depuis $1889-\mathrm{y}$ compris les lignes desservant les banlieues -, atteignait 153 kilomètres et transportait annuellement 157 millions de personnes. En 1896, on construisit le premier métro d'Europe et pendant la première guerre mondiale, les premiers autobus firent leur apparition dans les rues de Budapest. Les services communs municipalisés s'étendirent eux aussi considérablement : en 1901, le réseau principal d'alimentation en gaz atteignait 601 kilomètres et la consommation s'élevait à 63 millions de m3 par an. En 1910, la longueur du réseau de câbles électriques était de 505 kilomètres et, grâce à l'extension continuelle du service des eaux, cette même année, 73 millions de $\mathrm{m} 3$ furent livrés dans les maisons par des conduites principales d'une longueur de 265 kilomètres. L'écoulement en était assuré par un réseau d'égouts moderne. Grâce à des équipements, la santé publique s'améliora considérablement dans la ville, et la mortalité passa de 38,9 pour mille en $1874-1875$ à 16,1 pour mille en 1911-1912. Toutefois, il existait une grande différence entre les quartiers riches et les quartiers ouvriers.

Malgré cela, le rythme de la construction des logements et des services publics n'avait pas suivi le rythme d'accroissement de la population; en Europe, c'est à Budapest que les 
petits logements surpeuplés étaient en plus grand nombre et cela touchait principalement le prolétariat. Ainsi, entre 1880 et 1910, le nombre de locataires par chambre, même dans les quartiers du centre, ne diminua que de 2,91 à 2,54 tandis qu'il augmentait considérablement dans les quartiers périphériques passant de 2,63 à 3,31. Une partie des ouvriers, surtout ceux qui habitaient les banlieues, étaient massés dans des sous-sols et dans des asiles de nuit; d'autres s'installèrent en dehors de la ville, autour des usines de la grande industrie : ils y bâtirent des habitats de mauvaise qualité qui se développèrent avec une rapidité extraordinaire. Le premier Ujpest, fondé à la limite de la ville, commença à se former dans les années 1830. En 1870, au Sud de la rive gauche, ce fut le peuplement d'Erzsebetfalva, Kispest, Pestlörinc. Bientôt des habitats se créèrent aussi à proximité de villages paysans anciens, groupés surtout à l'Est de la ville, et qui avaient gardé leur structure économique et sociale traditionnelle; la croissance de ces habitats est bien mise en valeur par le fait qu'en 1900 les sept localités les plus importantes de la grande banlieue de Budapest comptaient 105000 habitants dont $61 \%$ travaillaient dans l'industrie. En dix ans, cette population atteignit 183000 habitants et à la fin de la première guerre mondiale, 240000 habitants.

A partir de 1893, la ville fut gérée par arrondissements. Du fait de cette situation, en 1908, quelques urbanistes progressistes élaborèrent le premier projet d'intégration des banlieues aux limites administratives de Budapest, conformément à l'évolution que connaissaient plusieurs grandes villes de l'Europe centrale et occidentale. Pourtant, la bourgeoisie de Budapest, qui tenait l'administration de la ville, refusa ce projet, craignant les énormes dépenses qu'une telle extension entraînerait mais surtout redoutant la diminution de son influence politique. Les références aux aspects matériels, à la situation financière de la ville, n'étaient pas dépourvues de fondement. La politique économique traditionnelle, également élaborée dans les années 1870, reposait surtout sur les impôts et les taxes, et avait entraîné un très lourd endettement sans proposition de solutions financières modernes. Pourtant, tant le développement de l'administration urbaine (dont les attributions et le personnel prenaient de plus en plus d'importance) que l'augmentation constante des dépenses d'urbanisation en rendaient urgente la définition. Ce n'est qu'au début des années 1900 qu'une politique économique adaptée aux besoins de la ville devenue si rapidement une métropole, fut mise en oeuvre. Pour assainir l'état des finances, des investissements à long terme furent entrepris; les services communautaires furent municipalisés et dirigés comme des entreprises. Parallèlement, une politique sociale spécifique fut mise en oeuvre: construction d'immeubles à appartements modestes, construction d'écoles, efforts de diffusion culturelle, etc. Pour y parvenir les nouvelles autorités municipales, qui étaient libérales - parmi elles figure le nom d'Istvan Barczy, le maire - s'allièrent avec le parti des petits bourgeois radicaux, en cours de formation et dirigé par Vilmos Vazsonyi, et fit même quelques pas en direction de la classe ouvrière en proposant d'étendre le droit de vote.

Dès le début $\mathrm{du} \mathrm{xx}^{\mathrm{e}}$ siècle et parallèlement à la croissance de la grande ville moderne, les conflits sociaux s'aggravèrent, souvent jusqu'à frôler l'explosion. A cette époque la grande bourgeoisie de la ville dirigeait déjà la quasi totalité de la vie économique du pays. Ses alliés étaient les hauts fonctionnaires ainsi que l'aristocratie qui, grâce à son alliance avec la grande bourgeoisie, conservait et même développait son influence politique traditionnelle. Les lois assuraient une influence toute particulière sur la ville à ces couches sociales qui comptaient les habitants les plus riches: la moitié du conseil 
municipal n'était élue que par les 1200 citadins les plus imposés. De ce fait, la politique urbaine périclita dès l'origine.

Les premiers mouvements politiques populaires importants se formèrent à la fin du XIX et au début $\mathrm{du} \mathrm{xx}^{\mathrm{e}}$ siècle, prenant appui sur l'essor de la petite bourgeoisie qui accompagna logiquement le développement économique. Ces diverses tendances, qui revendiquaient une politique plus progressiste tant en matière de problèmes urbains qu'à l'égard des problèmes touchant l'ensemble du pays, avaient un poids politique important en raison du droit de vote, défini depuis 1874 par un cens relativement peu élevé. Cependant, étant donné la répartition des revenus extrêmement polarisée de la société urbaine, ce droit de vote touchait à peine 8 à $9 \%$ de la population totale. Les éléments bourgeois, radicaux et petits bourgeois, qui réclamaient une politique progressiste, souhaitaient le suffrage universel. Ils trouvaient des alliés actifs auprès des effectifs croissants de la classe ouvrière urbaine. Après la dissolution de l'Alliance Générale des Ouvriers, en 1873, c'est la presse socialiste et l'Association Générale d'Assurance Maladie des ouvriers qui assurèrent la continuité du mouvement ouvrier. En 1876, Léon Frankel, Ministre du Travail de la Commune de Paris, originaire de Budapest, prit la direction du mouvement. En 1880, le Parti Général des Ouvriers de Hongrie fut fondé à Budapest. Après l'arrestation de Frankel en 1881 et son exil en 1883, le parti adopta une politique de compromis ; cependant, malgré ses erreurs et ses limites, son existence et ses activités préparèrent la formation à Budapest en 1890, avec l'aide de la $\mathrm{II}^{\mathrm{e}}$ Internationale, du Parti social-démocrate de Hongrie. La même année, les ouvriers célébrèrent le 1er Mai en défilant également dans la capitale. Pendant les années 1890, le mouvement syndical s'intensifia à la faveur de l'essor industriel : en 1899, le premier congrès des syndicats hongrois se tint à Budapest.

De façon constante la capitale est restée le centre le plus actif du mouvement, également en ce qui concerne l'opposition de gauche à la direction réformiste du parti. Il influença considérablement l'évolution de la politique sociale de la ville, aussi bien que la position (prudemment progressiste) de l'administration urbaine sur les questions politiques nationales. Le prolétariat de Budapest démontra sa force à l'occasion des manifestations de 1905, 1907 et 1912.

$19 \mathrm{Au}$ cours de cette période, Budapest, seule grande ville de la Hongrie, est également le centre quasi exclusif de la culture hongroise. La politique scolaire y réduisait l'analphabétisme. La plupart des institutions scientifiques, des universités, des collections publiques, les plus grandes maisons d'édition, les sociétés littéraires, artistiques et scientifiques y avaient leur siège; seule Budapest faisait autorité dans n'importe quel domaine de la vie culturelle. A partir de 1908, paraît à Budapest la revue Nyugat (Occident), organe le plus influent de la littérature hongroise moderne. C'est encore à Budapest que s'épanouit la culture théâtrale et musicale la plus diverse, offrant aux tendances artistiques progressistes des possibilités de s'épanouir à côté des genres en vogue dans la petite bourgeoisie, tels que l'opérette et les chansons. 